\title{
Free products of topological groups
}

\section{Sidney A. Morris}

In this note the notion of a free topological product $\prod^{*} G_{\alpha}$ of a set $\left\{G_{\alpha}\right\}$ of topological groups is introduced. It is shown that it always exists, is unique and is algebraically isomorphic to the usual free product of the underlying groups. Further if each $G_{\alpha}$ is Hausdorff, then $\prod^{*} G_{\alpha}$ is Hausdorff and each $G_{\alpha}$ is a closed subgroup. Also $\prod^{*} G_{\alpha}$ is a free topological group (respectively, maximally almost periodic) if each $G_{\alpha}$ is. This notion is then combined with the theory of varieties of topological groups developed by the author. For $\underline{\underline{V}}$ a variety of topological groups, the V-product of groups in $\underline{\underline{V}}$

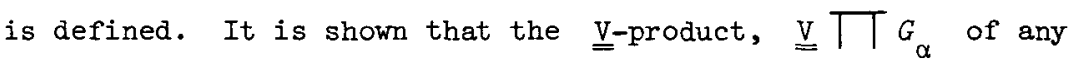
set $\left\{G_{\alpha}\right\}$ of groups in $\underline{V}$ exists, is unique and is algebraically isomorphic to the usual varietal product. It is noted that the $\underline{V}$-product of Hausdorff groups is not necessarily Hausdorff, but is if $\underline{\underline{V}}$ is abelian. Each $G_{\alpha}$ is a quotient

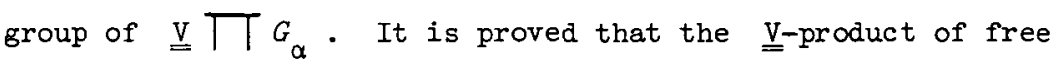
topological groups of $\underline{\underline{V}}$ and projective topological groups of $\underline{\underline{v}}$ are of the same type. Finally it is shown that $\underline{\underline{V}} \prod G_{\alpha}$ is connected if and only if each $G_{\alpha}$ is connected.

\section{Introduction}

In this note we introduce the notion of a free topological product of Received 12 August 1970. 
topological groups. The concept is the natural analogue of that of a free product of groups [5] and was inspired by the work of Golema [2] and Hulanicki [7] on free products in the category of compact Hausdorff groups.

In $\$ 2$ we prove basic existence and uniqueness theorems for the free topological product and the free abelian topological product and ape some theorems on free products. We note that Hulanicki's result (Proposition 1 of [7]) can be interpreted as saying the free topological product of compact Hausdorff groups is maximally almost periodic [15]. We extend this result to show that the free topological product of maximally almost periodic groups is of the same type. Hall [4] noted that the direct product of projective topological groups is not necessarily a projective topological group. In the light of this it is satisfying to see that the free abelian topological product of projective topological groups is projective.

In $\$ 3$ we combine the notion of a free topological product with that of a variety of topological groups; (see [10], [11] and [12]). We define a V-product of topological groups as a natural analogue of a varietal product

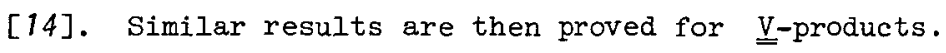

The notation and terminology, in general, conform to that of $[8]$ and [14]. Further if $G$ is a topological group then $\bar{G}$ denotes the underlying (abstract) group. If $S$ is any set then $|S|$ denotes the cardinal of the set. If $A$ and $B$ are topological groups then $A$ is a subgroup of $B$, means that $A$ is algebraically a subgroup of $B$ and that the topology induced on $A$ by $B$ is the given topology. The cartesian product of topological groups $\left\{G_{i}: i \in I\right\}$ is always given the Tychonoff product topology and denoted by $\prod_{i \in I} G_{i}$.

\section{Free topological products}

DEFINITION 2.1. Let $\left\{G_{i}: i \in I\right\}$ be a set of topological groups. Then the topological group $F$ is said to be a free topological product of $\left\{G_{i}: i \in I\right\}$, denoted by $\prod_{i \in I}^{*} G_{i}$, if it has the properties :

(a) for each $i$ in $I, G_{i}$ is a subgroup of $F$; 
(b) $F$ is generated algebraically by $\underset{i \in I}{\cup} G_{i}$;

(c) if for each $i$ in $I, \phi_{i}$ is a continuous homomorphism of $G_{i}$ into a topological group $H$, then there exists a continuous homomorphism $\Phi$ of $F$ into $H$ such that $\Phi=\phi_{i}$ on $G_{i}$, for each $i$.

THEOREM 2.2. Let $\left\{G_{i}: i \in I\right\}$ be any set of Hausdorff groups. Then $\prod_{i \in I}^{*} G_{i}$ exists cond is Housdorff.

Proof. Let $\bar{F}=\prod_{i \in I}{ }^{*} \bar{G}_{i}$ be the free (algebraic) product of the groups $\left\{\bar{G}_{i}: i \in I\right\}$. (See [5].) Our first task is to show that $\bar{F}$ can be given a Hausdorff group topology which induces the given topology on each $G_{i}$.

We define a topology $\tau_{1}$ on $\vec{F}$ by exhibiting a system of left-invariant pseudo-metrics $d$ ([8], p. 210) each of which is given by a family $\left\{d_{i}: i \in I\right\}$, where $d_{i}$ is a left-invariant pseudo-metric on $G_{i}$ for each $i$ in $I$.

For $g$ any element of $\bar{F}, g=g_{\alpha_{1}} \cdots g_{\alpha_{n}}$, where $g_{\alpha_{i}} \in G_{\alpha_{i}}$ and $\alpha_{i} \neq \alpha_{i+1}$. Define $d(g, e)=\sum_{i=1}^{n} d_{\alpha_{i}}\left(g_{\alpha_{i}}, e\right)$, where $e$ represents the identity element of $\bar{F}$ and each $G_{i}$. Then for $h$ and $k$ any elements in $\bar{F}$, define $d(h, k)=d\left(k^{-1} h, e\right)$. It is easily seen that $d$ is a left-invariant pseudo-metric on $\bar{F}$ and if $g$ is an element of $\bar{E}$ such that $d(g, e)=0$ for all $d$, then $g=e$. Thus the topology $\tau_{1}$ on $\vec{F}$ induced by the family of all $d$ is a Hausdorff group topology. Clearly $\tau_{1}$ induces the given topology on each $G_{i}$.

Now define $F$ to be the group $\bar{F}$ with the finest group topology $T$ which induces the given topology on each $G_{i}$. From the previous paragraph we see that $F$ exists and is Hausdorff. We claim that $F$ is the required 
group. (Clearly it has properties (a) and (b) of Definition 2.1.)

Let $H$ be any topological group and $\phi_{i}$, for each $i$ in $I$, be a continuous homomorphism of $G_{i}$ into $H$. Since $E$ is algebraically a free product of the $G_{i}$, there is a homomorphism $\Phi$ of $E$ into $H$ such that $\Phi=\phi_{i}$ on $G_{i}, i \in I$. Define a topology $\tau_{2}$ on $\bar{F}$ in the following manner: the set 0 is open in $\tau_{2}$ if and only if $0=\Phi^{-1}(A)$, where $A$ is an open set in $H$. Clearly $\tau_{2}$ is a group topology. Let the topology $\tau_{3}$ be the sum of $\tau_{2}$ and $\tau ;([3], \S 1)$. Then $\bar{F}$ with the topology $\tau_{3}$ is a topological group and $\tau_{3}$ induces the given topology on each $G_{i}$. Since $\tau_{3}$ is finer than $\tau$ we must have $\tau_{3}=\tau$. Hence $\Phi$ is continuous, and the proof is complete.

The next theorem shows that the free topological product is unique (if it exists). The proof is omitted as it is similar to that of Theorem 8.9 of [6].

THEOREM 2.3. Let $\left\{G_{i}: i \in I\right\}$ be a set of topological groups. If $F_{1}$ and $F_{2}$ both satisfy conditions (a), (b) and (c) of Definition 2.1, then there is a topological isomorphism $f$ of $F_{1}$ onto $F_{2}$ such that the mapping $f$ restricted to $G_{i}\left(\subseteq F_{1}\right)$ is a topological isomorphism of $G_{i}$ onto $G_{i}\left(\subseteq F_{2}\right)$ for each $i \in I$.

COROLLARY 2.4. If $\left\{G_{i}: i \in I\right\}$ is a set of Hausdorff groups, then $\prod_{i \in I}^{*} G_{i}$ is algebraically isomorphic to the free (algebraic) product of $\left\{\vec{G}_{i}: i \in I\right\}$.

THEOREM 2.5. Let $\left\{G_{i}: i \in I\right\}$ be a set of Hausdorff groups and $J$ be a subset of $I$. Then the subgroup $G$ of $\prod_{i \in I}^{*} G_{i}$ generated algebraically by $\underset{i \in J}{\cup} G_{i}$ is topologically isomorphic to $\prod_{i \in J}^{*} G_{i}$. Further, $G$ is a closed subgroup of $\prod_{i \in I}^{*} G_{i}$.

Proof. In the notation of Theorem 2.2, it is clear that $G$ is a 
closed subset in $\tau_{1}$. Since $\tau$ is finer than $\tau_{1}, G$ is a closed subgroup of $\prod_{i \in I}^{*} G_{i}$.

Let $H$ be any topological group and $\phi_{i}$ a continuous homomorphism of $G_{i}$ into $H$ for each $i$ in $J$. For each $i$ in $I$ which is not in $J$, define a continuous homomorphism $\phi_{i}$ of $G_{i}$ into $H$ by $\phi_{i}\left(G_{i}\right)=e$, the identity of $H$. Then there is a continuous homomorphism $\Phi$ of $\prod_{i \in I}^{*} G_{i}$ into $H$ such that $\Phi=\phi_{i}$ on $G_{i}$. Restrict $\Phi$ to $G$. Clearly then, condition (c) of Definition 2.1 is satisfied. Hence $G$ is the free topological product of $\left\{G_{i}: i \in J\right\}$.

COROLLARY 2.6. If $\left\{G_{i}: i \in I\right\}$ is a set of Hausdorff groups then each $G_{i}$ is a closed subgroup of $\prod_{i \in I}^{*} G_{i}$.

The following lema can be proved in precisely the same way as Lemma 2.11 of [10].

LEMMA 2.7. Let $X$ be any subset of a topological group $H$. If $\Phi$ is a homomorphism of $H$ into a topological group $G$ such that $\Phi \mid X$ is an open mapping, then $\Phi$ is an open mapping.

THEOREM 2.8. Let $\prod_{i \in I}^{*} G_{i}$ be the free topological product of a set $\left\{G_{i}: i \in I\right\}$ of topological groups. Then each $G_{i}$ is a quotient group of $\prod_{i \in I}^{*} G_{i}$.

Proof. Let $j$ be a fixed element of $I$. For each $i$ in $I$, $i \neq j$, define a mapping $\phi_{i}$ of $G_{i}$ into $G_{j}$ by $\phi_{i}\left(G_{i}\right)=e$. Define $\phi_{j}$ to be the identity mapping of $G_{j}$ into itself. Then each $\phi_{i}$, $i \in I$, is a continuous homomorphism. Therefore, there is a continuous homomorphism $\Phi$ of $\prod_{i \in I} T_{i} G_{i}$ into $G_{j}$ such that $\Phi \mid G_{i}=\phi_{i}$, for each $i$ in $I$. 
Now $\Phi \mid G_{j}$ is an open mapping. Thus by Lemma 2.7, $\Phi$ is an open mapping, and the proof is complete.

THEOREM 2.9. Let $F\left(X_{i}\right)$ be a free topological group on a topological space $X_{i}$, for $i$ in an index set $I$. ([9], or [10], Definition 4.1.) Then $\prod_{i \in I}^{*} F\left(X_{i}\right)$ exists and is $F(Y)$, the free topological group on the space $Y$, where $Y$ is the free union [1] of the spaces $\left\{X_{i}: i \in I\right\}$.

Proof. By Theorem 6.5 of [12], $F(Y)$ exists. It is easily verified that the subgroup of $F(Y)$ generated algebraically by $X_{i}$ is $F\left(X_{i}\right)$, for each $i$ in $I$.

Let $\phi_{i}$ be a continuous homomorphism of $F\left(X_{i}\right)$ into a topological group $H$, for each $i$ in $I$. Then $\phi_{i} \mid X_{i}$ is a continuous mapping of $X_{i}$ into $H$ for each $i$. Define a map $\phi$ of $y$ into $H$ by $\phi\left|X_{i}=\phi_{i}\right| X_{i}$, for each $i$. Then $\phi$ is continuous. Thus there is a continuous homomorphism $\Phi$ of $F(Y)$ into $H$ such that $\Phi \mid Y=\phi$. Hence $\Phi \mid X_{i}=\phi_{i}$ and therefore $\Phi \mid F\left(X_{i}\right)=\phi_{i}$, for each $i$. Thus $F(Y)$ is $\prod_{i \in I}^{*} F\left(X_{i}\right)$

DEFINITION 2.10. A topological group $G$ is said ta be maximally almost periodic (MAP), if it admits a continuous monomorphism into a compact Hausdorff group; (see [15]).

For example every compact Hausdorff group, and every locally compact Hausdorff abelian group is a MAP group.

THEOREM 2.11. If $\left\{G_{i}: i \in I\right\}$ is a set of MAP groups, then $\prod_{i \in I}^{*} G_{i}$ is a MAP group.

Proof. For each $i$ in $I$, there is a compact Hausdorff group $H_{i}$ and a continuous monomorphism $\phi_{i}$ of $G_{i}$ into $H_{i}$. Proposition 1 of 
[7] implies that $\prod_{i \in I}^{*} H_{i}$ is a MAP group.

Furthermore there is a continuous homomorphism of $\prod_{i \in I}^{*} G_{i}$ into $\prod_{i \in I}^{*} H_{i}$ such that $\Phi \mid G_{i}=\phi_{i}$, for each $i$. Clearly $\Phi$ is a monomorphism. Hence $\prod_{i \in I}^{*} G_{i}$ is a MAP group.

REMARK 2.12. If we restrict our attention to abelian groups we can obtain similar results. In fact if we take all groups previously discussed in this section to be abelian (and hence define a free abelian topological product) all the results remain true.

The following theorem is obvious.

THEOREM 2.13. If $\left\{G_{i}: i \in I\right\}$ is any set of abelian topological groups then their free abelian topological product exists and has the direct product $\prod_{i \in I}^{D} G_{i}$ of these groups as a continuous monomorphic image.

REMARK 2.14. Remark 3.32 of [13] states that the direct product of free abelian topological groups is not necessarily a free abelian topological group. Thus the analogue for abelian groups of Theorem 2.9, which states that the free abelian topological product of free abelian topological groups is a free abelian topological group, is of interest.

Also Theorem 2.15 is notable, since Example 2 of [4] shows that the direct product of projective topological groups is not necessarily projective.

THEOREM 2.15. Let $\left\{G_{i}: i \in I\right\}$ be a set of projective topological groups (in the sense of [4]). Then the free abelian topological product $F$ of $\left\{G_{i}: i \in I\right\}$ is a projective topological group.

Proof. Let $A$ and $B$ be abelian topological groups and $f$ be a continuous homomorphism of $A$ onto $B$ such that there exists a continuous mapping $g$ of $B$ into $A$ such that $f g$ acts identically on $B$. 
Let $\theta$ be a continuous homomorphism of $F$ into $B$ and $\theta_{i}=\theta \mid G_{i}$ for each $i$ in $I$. Then $\theta_{i}$ is a continuous homomorphism of $G_{i}$ into $B$. Since each $G_{i}$ is projective, there is a continuous homomorphism $\phi_{i}$ of $G_{i}$ into $A$ such that $f \phi_{i}=\theta_{i}$. Therefore there is a continuous homomorphism $\Phi$ of $F$ into $A$ such that $\left.\Phi\right|_{i}=\phi_{i}$. Clearly $f \Phi=\theta$. Hence $F$ is projective.

\section{Topological varietal products}

DEFINITION 3.1. Let $\underline{\mathrm{V}}$ be a variety of topological groups [10] and $\left\{G_{i}: i \in I\right\}$ be a set of topological groups in $\underline{\underline{V}}$. Then the topological

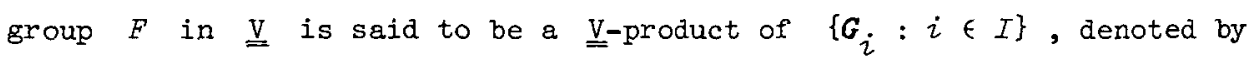
$\underline{V} \prod_{i \in I} G_{i}$, if it has the properties:

(a) each $G_{i}, i \in I$, is a subgroup of $F$;

(b) $F$ is generated algebraically by $\underset{i \in I}{U} G_{i}$;

(c) if for each $i$ in $I, \phi_{i}$ is a continuous homomorphism of $G_{i}$ into a topological group $H$ in $\underline{\underline{V}}$, then there exists a continuous homomorphism $\Phi$ of $F$ into $H$ such that $\Phi=\phi_{i}$ on $G_{i}$, for each $i$.

Clearly, if $\underline{\underline{V}}$ is the variety of all (respectively, all abelian) topological groups, then $\underline{V} \prod_{i \in I} G_{i}$ is the free (free abelian) topological product of $\left\{G_{i}: i \in I\right\}$.

THEOREM 3.2. Let $\left\{G_{i}: i \in I\right\}$ be any set of topological groups in a variety of topological groups $\underline{\underline{V}}$. Then $\underline{\underline{v}} \prod_{i \in I} G_{i}$ exists.

Proof. Let $m$ be any infinite cardinal such that $\left|\underset{i \in I}{U} G_{i}\right| \leq m$. Let $\left\{H_{\alpha}: \alpha \in M\right\}$ be the set of all (non-topologically isomorphic) topological groups in $\underline{V}$ such that $\left|H_{\alpha}\right| \leq m$. We denote by $\beta$ a system 
of continuous homomorphisms $f_{i}^{\alpha \beta}, i \in I$ of the $G_{i}$ into a fixed $H_{\alpha}$. The set of all such systems may be denoted by $N_{\alpha}$. Let $G=\prod_{\alpha, \beta} H_{\alpha \beta}$, with $H_{\alpha \beta}=H_{\alpha}$ for $\beta \in N_{\alpha}$.

For each $i$ in $I$, define the map $\sigma_{i}$ of $G_{i}$ into $G$ by $\sigma_{i}(x)=\left\{f_{i}^{\alpha \beta}(x)\right\}$. Clearly by the embedding lemma in [8], each $\sigma_{i}$ is a topological isomorphism of $G_{i}$ onto $\sigma_{i}\left(G_{i}\right)$. We now identify $G_{i}$ with $\sigma\left(G_{i}\right)$

Let $F$ be the subgroup of $G$ generated algebraically by $\underset{i \in I}{U} G_{i}$. Then $F$ is the required group. We see that $F$ has properties $(a)$ and (b) of Definition 3.1.

Let $\phi_{i}$ be a continuous homomorphism of $G_{i}$ into a topological group $H$ in $\underline{\underline{V}}$ for each $i$ in $I$. Without loss of generality we can assume $|H| \leq m$. Thus for some $\alpha$ and $\beta$ we can identify $H$ with $G_{\alpha \beta}$ and $\phi_{i}$ with $f_{i}^{\alpha \beta}$ for each $i$. Hence, if we define $\Phi$ to be the projection of $F$ onto the axis corresponding to the indices $\alpha, \beta$ we get $\Phi \mid G_{i}=\phi_{i}$, for each $i$ in $I$. The proof is complete.

The following uniqueness theorem can be proved in a similar manner to Theorem 8.9 of [6].

THEOREM 3.3. Let $\left\{G_{i}: i \in I\right\}$ be a set of topological groups in a variety of topological groups $\underline{\underline{\mathrm{V}}}$. If $F_{1}$ and $F_{2}$ are in $\underline{\underline{\mathrm{V}}}$ and have the properties stated in Definition 3.1, then there is a topological isomorphism $f$ of $F_{1}$ onto $E_{2}$ such that the mapping $f$ restricted to $G_{i}\left(\subseteq F_{1}\right)$ is a topological isomorphism of $G_{i}$ onto $G_{i}\left(\subseteq F_{2}\right)$ for each $i$.

THEOREM 3.4. Let $\underline{\underline{\mathrm{v}}}$ be a variety of topological groups and $\underline{\underline{\mathrm{V}}}$ be the underlying (algebraic) variety of groups. (See Remark 2.3 of [10].) If $\left\{G_{i}: i \in I\right\}$ is a set of topological groups in $\underline{\underline{\mathrm{V}}}$, then $\overline{\mathrm{V}} \prod_{i \in I} G_{i}$ 
is the $\underline{\underline{\mathrm{V}}}$-product of $\left\{\bar{G}_{i}: i \in I\right\}$. (See [14], 18.31.)

Proof. For each $i$ in $I$, let $\phi_{i}$ be a homomorphism (not necessarily continuous) of $G_{i}$ into any $H$ in $\underline{\underline{V}}$. It is enough to prove that there is a homomorphism $\Phi$ of $\underline{\underline{V}} \prod_{i \in I} G_{i}$ into $H$ such that $\Phi \mid G_{i}=\phi_{i}$ for each $i$

By Lemma 2.7 of [10], there is an $A$ in $\underline{\underline{V}}$ which has the indiscrete topology and is algebraically isomorphic to $H$. Let $\theta$ be an isomorphism of $H$ onto $A$. Then for each $i$, the mapping $\theta \phi_{i}$ is a continuous homomorphism of $G_{i}$ into $A$. Therefore, there is a continuous homomorphism $\delta$ of $\underline{V} \prod_{i \in I} G_{i}$ into $A$ such that $\delta \mid G_{i}=\theta \phi_{i}$, for each $i$. Define the mapping $\Phi$ of $\underline{\underline{\mathrm{V}}} \prod_{i \in I} G_{i}$ into $H$ by $\Phi=\theta^{-1} \delta$. Clearly $\Phi$ is a homomorphism and $\Phi \mid G_{i}=\phi_{i}$ for each $i$.

REMARK 3.5. Theorem 2.2 leads us to ask: If $\left\{G_{i}: i \in I\right\}$ is a set of Hausdorff groups in a variety of topological groups $\underline{\underline{V}}$, is $\underline{V} \prod_{i \in I} G_{i}$ necessarily Hausdorfe? The following example answers this question in the negative, whilst Theorem 3.7 shows that if $\underline{\underline{V}}$ is an abelian variety then the answer is positive.

EXAMPLE 3.6. (See [12], Example 3.2.) Let $\underline{\underline{v}}$ be the class of all topological groups $G$ having the property that the intersection of all neighbourhoods of the identity in $G$ contains the commutator subgroup of $G$. It can be readily verified that $\underline{\underline{V}}$ is a variety of topological groups such that the underlying (algebraic) variety of groups is the variety of all groups.

Clearly the infinite cyclic group $F$ with the discrete topology is

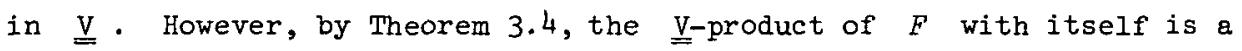
non-abelian group and hence is not Hausdorff.

THEOREM 3.7. Let $\underline{\mathrm{V}}$ be any variety of abelian topological groups and $\left\{G_{i}: i \in I\right\}$ be any set of Hausdorff groups in $\underline{\underline{\mathrm{V}}}$. Then $\underline{\underline{\mathrm{V}}} \prod_{i \in I} G_{i}$ 
is a Hausdorff group and each $G_{i}$ is a closed subgroup.

Proof. This follows immediately from the fact that the direct product of $\left\{G_{i}: i \in I\right\}$ is in $\underline{V}$, is a Hausdorff group which is algebraically isomorphic to $\mathrm{v} \prod_{i \in I} G_{i}$, and has each $G_{i}$ as a closed subgroup.

The next theorem is obvious.

THEOREM 3.8. Let $\underline{\underline{\mathrm{V}}}$ be any variety of topological groups and $\left\{G_{i}: i \in I\right\}$ a set of topological groups in $\underline{\underline{\mathrm{V}}}$. Consider the sequence

$$
\prod_{i \in I}^{*} G_{i} \stackrel{f}{\longrightarrow} \underline{\underline{v}} \prod_{i \in I} G_{i} \stackrel{g}{\rightarrow} \prod_{i \in I}^{D} G_{i}
$$

where $\prod_{i \in I}^{D} G_{i}$ is the direct product of the $G_{i}$ and the mappings $f$ and $g$ are the natural homomorphisms. Then $f$ and $g$ are continuous. Further, if $\mathrm{V}$ is a full variety [11], $f$ is an open mapping.

Theorems $3.9,3.10,3.11$ and 3.12 can be proved in a similar manner to Theorems $2.8,2.9,2.15$ and 2.5 respectively.

THEOREM 3.9. Let $\underline{V}$ be a variety of topological groups and $\left\{G_{i}: i \in I\right\}$ be in $\underline{V}$. Then each $G_{i}$ is a quotient group of $\underline{\underline{\mathrm{V}}} \prod_{i \in I} G_{i}$.

THEOREM 3.10. Let $\underline{\underline{\mathrm{V}}}$ be a variety of topological groups and $F\left(X_{i}, \underline{V}\right), i \in I$, be a set of free topological groups of $\underline{\underline{V}}[10]$. Let $Y=\bigcup_{i \in I} X_{i}$ in $\underline{\underline{\mathrm{v}}} \prod_{i \in I} F\left(X_{i}, \underline{\underline{\mathrm{v}}}\right)$. Then $\underline{\underline{\mathrm{v}}} \prod_{i \in I} F\left(X_{i}, \underline{\underline{\mathrm{v}}}\right)$ is $F(y, \underline{\underline{\mathrm{v}}})$.

THEOREM 3.11. Let $\underline{\underline{\mathrm{V}}}$ be a variety of topological groups and $\left\{G_{i}: i \in I\right\}$ be a set of topological groups projective in $\mathrm{V}$ [11]. Then $\underline{\mathrm{V}} \prod_{i \in I} G_{i}$ is projective in $\underline{\mathrm{v}}$.

Note that Theorem 3.6 of [11] is a special case of Theorem 3.11 .

THEOREM 3.12. Let $\left\{G_{i}: i \in I\right\}$ be a set of topological groups in 
a variety of topological groups $\underline{\underline{\mathrm{V}}}$. Let $J$ be a subset of $I$. Then the subgroup of $\underline{\underline{V}} \prod_{i \in I} G_{i}$ generated algebraically by $\underset{i \in J}{U} G_{i}$ is topologically isomorphic to $\underline{\underline{\mathrm{V}}} \prod_{i \in J} G_{i}$.

THEOREM 3.13. Let $\underline{\underline{\mathrm{V}}}$ be a variety of topological groups and $\left\{G_{i}: i \in I\right\}$ be in $\underline{\underline{\mathrm{V}}}$. Then $\underline{\underline{\mathrm{V}}} \prod_{i \in I} G_{i}$ is a connected group if and on $z y$ if each $G_{i}$ is connected.

Proof. If $\underline{\underline{V}} \prod_{i \in I} G_{i}$ is connected, then it follows immediately from Theorem 3.9 that each $G_{i}$ is connected.

Conversely if each $G_{i}$ is connected, then $\underset{i \in I}{\bigcup} G_{i}$ is a connected subset of $\underline{\underline{\mathrm{V}}} \prod_{i \in I} G_{i}$ containing the identity. Further $\underset{i \in I}{U} G_{i}$ generates $\underline{\mathrm{V}} \prod_{i \in I} G_{i}$ algebraically. Hence $\underline{\mathrm{V}} \prod_{i \in I} G_{i}$ is connected.

\section{References}

[1] James Dugundji, Topology (Allyn and Bacon, Boston, 1966).

[2] K. Golema, "Free products of compact general algebras", Colzoq. Math. 13 (1965), 165-166.

[3] M.l. Graev, "Free topological groups", Izv. Akad. Nauk SSSR Ser. Mat. 12 (1948), 279-324, (Russian). English transl., Amer. Math. Soc. Trans 2. no. 35, (1951). Reprint Amer. Math. Soc. Trans 2. (1) 8 (1962), 305-364.

[4] C.E. Hall, "Projective topological groups", Proc. Amer. Math. Soc. 18 (1967), 425-431.

[5] Marshall Hall, Jr, The theory of groups (The Macmillan Company, New York, 1959).

[6] Edwin Hewitt and Kenneth A. Ross, Abstract harmonic analysis, Vol. I (Die Grundlehren der mathematischen Wissenschaften, Band 115; Springer-Verlag, Berlin, Göttingen, Heidelberg, 1963). 
[7] A. Hulanicki, "Isomorphic embeddings of free products of compact groups", Colzoq. Math. 16 (1967), 235-241.

[8] John L. Kelley, General topology (Van Nostrand, Toronto, New York, London, 1955).

[9] A.A. Markov, "On free topological groups", C.R. (Doklady) Acad. Sci. URSS, (N.S.) 31 (1941), 299-301. Buzl. Acad. Sci. URSS Sér. Math. [Izv. Adak. Nauk SSSR] 9 (1945), 3-64. (Russian. English summary). English Transl., Amer. Math. Soc. Transl. no. 30 (1950), 11-88; reprint Amer. Math. Soc. Trans 2. (1) 8 (1962), 195-272.

[10] Sidney A. Morris, "Varieties of topological groups", BulZ. Austral. Math. Soc. I (1969), 145-160.

[11] Sidney A. Morris, "Varieties of topological groups II", BuzZ. Austral. Math. Soc. 2 (1970), 1-13.

[12] Sidney A. Morris, "Varieties of topological groups III", BuZZ. Austral. Math. Soc. 2 (1970), 165-178.

[13] Sidney A. Morris, "Varieties of topological groups", Ph.D. thesis, The Flinders University of South Australia, February 1970. [Abstract: Bull. Austral. Math. Soc. 3 (1970), 429-431.]

[14] Hanna Neumann, Varieties of groups (Ergebnisse der Mathematik und ihrer Grenzgebiete, Band 37, Springer-Verlag, Berlin, Heidelberg, New York, 1967).

[15] J. v. Neumann, "Almost periodic functions in a group. I", Trans. Amer. Math. Soc. 36 (1934), 445-492.

University of Adelaide, Adelaide, South Australia, and University of Florida, Gainesville, Florida, USA. 